\title{
Myxoid liposarcoma of hypopharynx- A diagnostic and management dilemma
}

\author{
Ravneet R. Verma ${ }^{1}$, Ravinder Verma ${ }^{2, *}$ \\ ${ }^{1}$ Senior Resident, Government Medical College and Hospital, Chandigarh ${ }^{2}$ Senior Surgeon, Dept. of ENT/Head and Neck, Verma \\ Hospital and Research Centre, Gujral Nagar, Jalandhar, Punjab, India \\ *Corresponding Author: \\ Email: verma1999@gmail.com
}

\begin{abstract}
Sarcomas are malignant neoplasms arising from connective tissue. They represent roughly $1 \%$ of all cancer cases reported. Overall, only 4-9\% of all liposarcomas occur in head and neck region. A small uncommon group of soft tissue tumours are distinguished by their unique and consistant ability to produce an overwhelming abundance of myxoid ground substance along with the proliferating cells that constitute the tumour. Grossely all these neoplasms have a variable gelatinous quality. Because of the voluminous stroma most of these tumours have some findings that overlap with one another. Nonetheless each tumour has a composite set of morphologic, immunophenotypic, ultrastructural and genotypic features exclusive to itself. ${ }^{26}$ (Wakely 1999) Hypopharyngeal localization is rarely described in the literature. Golledge et a1 ${ }^{11}$ (1995) reviewed the literature and reported 76 cases of liposarcoma of head and neck including 4 cases of their own. 38\% of liposarcomas of head and neck involve larynx and /or pharynx.78\% of these laryngeal pharyngeal cases arise in the supra glottic region and were well differentiated. Han et a $1^{13}$ (2014) reported a total of 37 cases of Laryngeal liposarcoma reported in English or French literature. Although more common in other areas, it is rarely found in the aero-digestive tract. Liposarcomas typically occur in middle aged to older adults. These tumors are typically misdiagnosed secondary to their indolent, asymptomatic course and similarities in appearance to other benign lesions. An understanding of these lesions will help clinicians appropriately manage their patients.
\end{abstract}

Keywords: Liposarcoma, Well-differentiated liposarcoma, Hypopharynx.

\section{Introduction}

Sarcomas are malignant neoplasms arising from connective tissue. They represent roughly $1 \%$ of all cancer reported .Overall, only $4-9 \%$ of all liposarcomas occur in the head and neck region. Hypopharyngeal localization is rarely described in the literature. Primary liposarcoma of the hypopharynx (pyriform sinus) is extremely rare. Only 20 case were reported in literature. ${ }^{6}$

Golledge et al $(1995)^{11}$ reviewed the literature and reported 76 cases of liposarcoma of head and neck including 4 cases of their own.38\% of liposarcomas of head and neck involve larynx and/or pharynx.78\% of these laryngeal/pharyngeal cases arise in the supraglottic region and were well differentiated. Han et $\mathrm{al}^{13}$ reported a total of 37 cases of Laryngeal liposarcoma reported in English and French literature. A small, uncommon group of these soft tissue tumors are distinguished by their unique and consistent ability to produce an overwhelming abundance of myxoid ground substance along with the proliferating cells that constitute the tumor. Grossly, all these neoplasms have a variable gelatinous quality. Because of the voluminous stroma, most of these tumors have some findings that overlap with one another. Nonetheless, each tumor has a composite set of morphologic, immunophenotypic, ultrastuctural and genotypic features exclusive to itself. ${ }^{26}$ Although more common in other areas, it is rarely found in the aerodigestive tract. Histologically, liposarcomas are divided in to four following subtypes: well differentiated, myxoid, round cells and pleomorphic. The well-diffrentiated subtype is further subdivided into three type of lipoma: sclerosing, inflamentory and undifferentiated. Liposarcomas typically occur in middle aged to older adults. These tumors are typically misdiagnosed secondary to their indolent, asymptomatic course and similarities in appearance to other benign lesions. An understanding of these lesions will help clinicians appropriately manage their patients.

Principal symptoms are dysphagia, dyspnea. dysphonia, airways obstruction and sensation of a foreign body. Treatment of choice is surgery and the literature describes the performance of lateral pharyngotomy, simple excision and even total laryngectomy. Previous studies have demonstrated that liposarcomas of the hypopharynx are well-differentiated and more commonly present as polypoid or exophytic tumors. Although they do not metastasize regionally, multiple recurrences are frequent. We present a case of recurrent liposarcoma of the hypopharynx treated with resection using suspension laryngoscopy and trans-oral laser.

\section{Case Report}

22 year old Male patient, came to us in 2010 with history of difficulty in breathing and swallowing. He had change in voice and repeated episodes of choking. $\mathrm{He}$ experienced stridor and sleep apnoea. On examination, he was found to have a mass in the hypopharynx. CT scan neck showed a smooth mass in $\mathrm{He}$ was advised surgery with preoperative tracheostomy. He did not agree for tracheostomy. $\mathrm{He}$ 
reported after a week with severe respiratory distress. An emergency tracheostomy was done under local anesthesia with supplemented oxygen. Under GA through tracheostomy, laryngoscopy was done. Smooth lobulated mass filling the right pyriform fossa and covering the laryngeal inlet was found. The mass was attached to Right aryepiglottic fold and adjoining medial wall of pyriform fossa. Anterior pharyngotomy was done. Mass looked fleshy, soft and lobulated with intact overlying mucosa. The three lobules were enucleated submucosally. Redundant mucosa excised and stitched. The wound closed in layers with nasogastric feeding tube. The patient discharged on $7^{\text {th }}$ Post operative day with closure of tracheostomy and oral feed started. The histopathology was reported to be Myxoid lipoma. ${ }^{28}$

In 2015, he came back with recurrence and similar symptoms and signs. Tracheostomy and anterior pharyngotomy with removal of the mass was done. The histopathology revealed it as Myxoid Lipoma with advice to get the IHC done. But patient refused and did not turn up for follow up.

In 2017 again, he reported with change in voice, difficulty in swallowing, difficulty in breathing with apoenic spells for the last three months. Endolaryngoscopic examination revealed a rounded mass filling the right pyriform sinus. (Fig. 1) CT scan neck revealed a heterogeneously enhancing polypoidal mass lesion causing effacement of right pyriform sinus involving right aryepiglottic fold with effacement of paralaryngeal fat. (Fig. 2) Trans oral removal of the mass was done using laser and cutting diathermy.Feeding nasogastric tube and tracheostomy was avoided. The patient was discharged on third postoperative day with oral feed and no respiratory distress. Taking into account of the recurrence, histopathology and immunohistochemistry were done. Microscopic examination shows myxoid spindle cell neoplasm with prominent chicken wire vasculature with monomorphic stellate to fusiform cells with minimal atypia. Lymphangioma like cystic changes were seen. Mitosis noted as 2-5/10 high power fields. Tumour giant cells were seen with few interspersed mature adipocytes. (Fig. 3)

CDK4 (EP180), (Fig. 4) S 100 (EP32) (Fig. 5) and MDM2 (Fig. 6) were positive. Ki67 (MIB-1) proliferation index was 2-5\%. (Fig. 7) Histopathology and IHC confirmed it to be well differentiated Myxoid Liposarcoma.

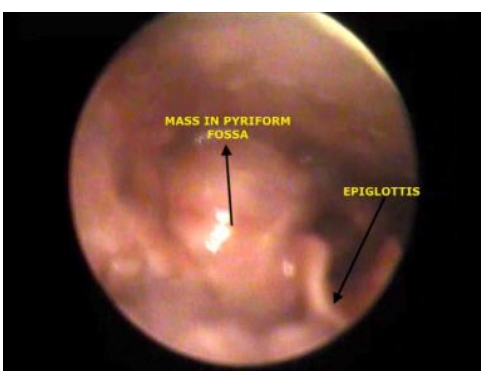

Fig. 1

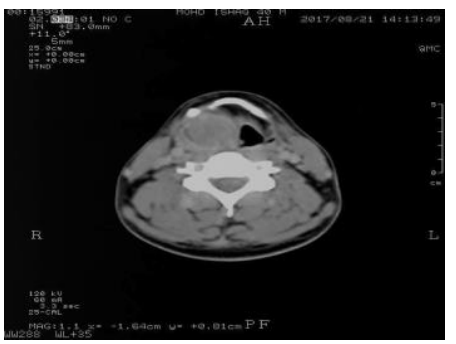

Fig. 2

Fig. 3

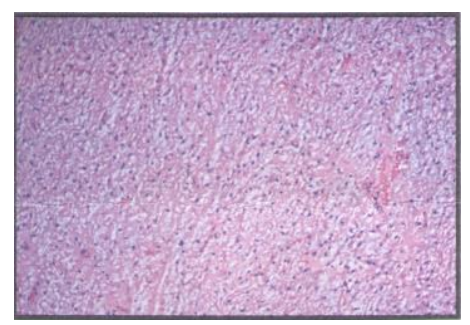

Fig. 4
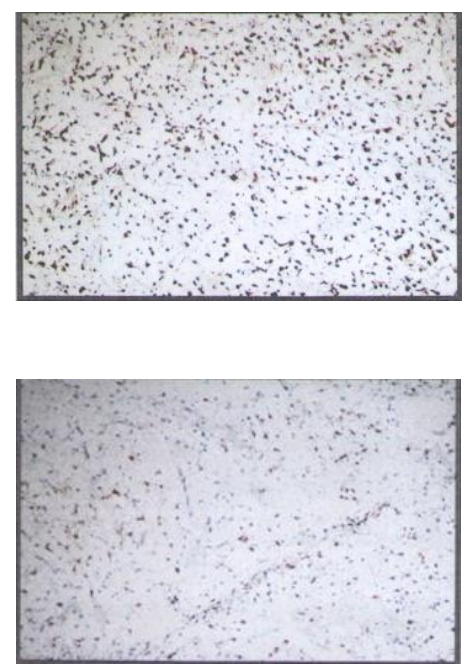

Fig. 5

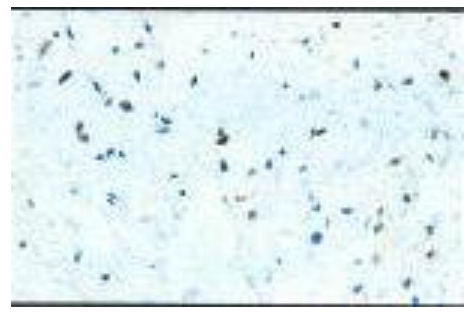

Fig. 6 


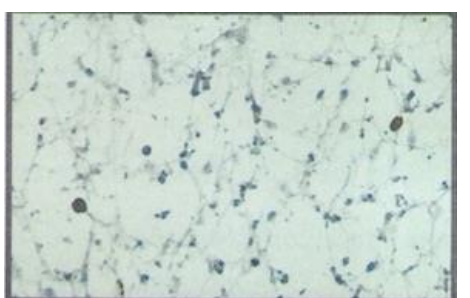

Fig. 7

\section{Discussion}

R. Virchow originally described Liposarcoma in 1857. In 1944, Arthur Purdy Stout wrote,"Surely, one of the most bizarre and fantastic chapters in the story of oncology in furnished by the tumors of fat-forming cells. The strange way in which they grow, their astounding size and many other peculiar features make them of great interest. ${ }^{9}$ A liposarcoma is a malignant mesenchymal neoplasm arising from deep-seated adipose tissue. Although rare, there have been reports of these neoplasms arising from pre-existing lipomas. Liposarcomas are the second most comman soft tissue sarcoma. They represent roughly $9-18 \%$ of all sarcomas and tend to have a high predilection for the extremities and retroperitoneum. Overall, only 4-9\% of all liposarcomas occur in the head and neck region. ${ }^{2}$ Hypopharyngeal localization is rarely described in the literature. Golledge et al in $1995^{11}$ reviewed the literature and reported 76 cases of liposarcoma of head and neck including 4 cases if their own. 38\% of liposarcomas of head and neck involve larynx and /or pharynx.78\% of these laryngeal/pharyngeal cases arise in the supraglottic region and were differentiated. Mandell et $\mathrm{al}^{18}$ in 1999 had reviewed 39 cases of liposarcoma of upper aerodigestive tract, among which 26 were located in the larynx, ${ }^{7}$ in the hypopharynx, and 6 in the oesophagus. Han et $\mathrm{al}^{13}$ reported a total of 37 cases of Laryngeal liposarcoma reported in English or French literature. Wenig et al in $1990^{27}$ reported that tumor occurred in patients ranging in age 37 to 77 years with marked male to female predominance of 9 to 1 . The tumors, which occurred exclusively in the supraglottic larynx or hypopharynx (pyriform sinus) caused airways obstruction; this was the most common presenting symptom. Atypical cells, scattered lipoblasts and infiltration differentiated malignant laryngeal fatty tumors from benign ones. Their occurrence in children is very rare accounting for less than 5\% of all soft tissue sarcoma cases in children. ${ }^{15,23}$

Liposarcomas are classified by their histopathological appearance. WHO(2013) classified soft tissue tumors. There are four recognized subtypes : well-differentiated, myxoid/round-cell, dedifferentiated, and pleomorphic. The myxoid subtype is the most common subtype, representing anywhere from $45-55 \%$ of cases. These tumors are considered low to intermediate grade. The well-differentiated subtype is the second most common subtype and represent 30$40 \%$ of cases. It is the lowest grade of all subtype, and as such, tends to carry a better prognosis. The welldifferentiated liposacorma is divided into three subtype, with the lipoma-like liposacorma-the most common mimicking the lipoma both macroscopically and microscopically. The other two subtypes are inflammatory and sclerosing liposarcomas. The roundcell subtype is considered the high-grade counterpart of the myxoid subtype and typically represent $15 \%$ of all cases of liposarcomas. The dedifferentiated subtype is believed to arise from long standing or recurrent welldifferentiated tumors. Depending on the amount of dedifferentiated, these can be intermediate or high grade. The pleomorphic subtype is the highest-grade subtype, and caries the worst prognosis. Fortunately, this subtype is rare, accounting for only 5\% of all liposarcoma cases. ${ }^{2,9}$ Doyle, ${ }^{5}$ in his review article described myxoid liposarcoma graded as low, intermediate or high grade, based on degree of cellularity.

The aetiology of soft tissue tumours is unknown. The development of a liposarcoma from a pre-existing benign lipoma is rare. Rarely they are associated with genetics and environmental factors, irradiation, viral and immune deficiencies. Majority of these tumours arise de novo without any causative factor. ${ }^{7}$ Liposarcomas most frequently arise from the deepseated stroma rather than submucosal or subcutaneous fat. The pathogenesis behind these neoplasms is believed to involve various chromosomal abnormalities. Fusion proteins created by chromosomal abnormalities are key components of mesenchymal cancer development. An abnormality of band $12 \mathrm{q} 13$ has been associated with the development of liposarcomas. The most common chromosomal translocation is the FUSCHOP fusion gene, which encodes a transcription factor necessary for adipocyte differentiation. These and other distinct genetic aberrations may aid in the diagnosis of particular liposarcoma subtype and they can potentially be targets that can be exploited therapeutically ${ }^{1}$ The diagnosis of a liposarcoma is typically delayed secondary to its indolent, asymptomatic course. Myxoid tumours of soft tissue encompass a heterogeneous group of lesions, characterized by a marked abundance of extracelular mucoid (myxoid) matrix. This group of tumours demonstrates significant variability in their biological behavior thus including tumours, which are entirely harmless, with a tendency to recur locally but not metastasize and malignant tumours. There appears to be a considerable degree of overlap clinically and morphologically between the various tumour types in this group, generating potential diagnostic problems for the clinician and pathologist alike. While dilligent microscopy remains the basis of diagnostic pathology, the continuous developments and refinements within the field of immunohistochemistry and molecular cytogenetics are providing substantial new information, allowing the development of new diagnostic criteria 
and hence facilitating an accurate diagnosis. ${ }^{12}$ Upon gross inspection, these tumours appear very similar to those of lipomas. However, there are details that help to differentiate the two. Liposarcomas tend to be more fixed to the underlying tissue, less compressible, and firmer to palpation .They also tend to be less lobulated and less yellow in color when compared to lipomas. There are multiple imagining modalities available to aid the clinician in diagnosing liposarcomas. Of these, the most commonly utilize are the MRI and CT scans. The MRI is probably the most useful since it may reveal characteristics of the tumor that are suggestive of malignancy. On MRI, liposarcomas appear well circumscribed and lobulated with thick, fibrous septaehypointense in T1-and T2-weighted images and enhanced after intravenous gadolinium injection. The fat in the other types of liposarcomas is arranged in a mesh-like pattern or in linear or nodular clusters. Intramuscular liposarcomas show nodularity. In addition to these findings, the presence of hemorrhage, edema and areas of necrosis may also be noted, which may hint at malignancy. The level of enhancement can also be utilized in assessing these tumors. Little enhancement is noted in well-differentiated liposarcomas, and more enhancement is seen with more aggressive round cell, pleomorphic and dediffentiated subtypes. ${ }^{3}$ Other findings characteristic of liposarcoma are thick fibrous septae, nodularity and contrast enhancement on fat-suppressed sequences. ${ }^{21}$

The amount of heterogeneity is also important as it can determining the grade of the tumor. Welldifferentiated liposarcomas typically appear homogeneous with more intense fat signal while myxoid types exhibit slight heterogeneity. The dedifferentiated, round cell and pleomorphic types tend to exhibit moderate to marked heterogeneity. ${ }^{9}$ The gold standard in diagnosing a liposarcoma remains the biopsy. This can be done through fine needle aspiration and incisional or excisional biopsy. Some recommend avoidance of excisional biopsy in suspected cases of liposarcoma as it may negatively affect treatment planning. Most atypical lipomatous tumours can be diagnosed microscopically based in H\&E sections, sometimes it may be difficult to make the differential diagnosis between ALT and lipoma or between ALT and spindle cell/pleomorphic lipoma. In these cases, immunoshistochemical staining if MDM-2 and CDK4 may be very useful as an aid in the differential diagnosis. ${ }^{25}$

While each other subtype has its own pathological characteristics, the key to the diagnosis is the presence of the lipoblast. Three main features characterize these tumors. The first is the presence of multiple lipoblasts in various stages of development. The second feature is the delicate plexiform capillary network. Finally, the stromal matrix is composed of hyaluronidase-sensitive acid mucopolysaccharides. The least cellular forms are considered lower grade tumors, and typically contain more spindle-shaped cells. The high cellular firms are considered high grade and typically exhibit an abundance or round cells.

Although surgery remains the principal therapeutic modality in soft tissue sarcoma, the extent of surgery required, along with the optimum combination of radiotheraphy and chemotheraphy, remains controversial. In designing a treatment plan, the multidisciplinary team must balance the goal of minimizing local and distant recurrence with the aim of preserving function and quality of life. A properly executed surgical resection remains the most important part of the overall treatment. In general, the scope of the excision is dictated by the size of the tumors, its anatomical relation to normal structures (e.g. major neurovascular bundles) and the degree of function that would be lost after operation. If severe loss of function is likely, the key question is whether this can be minimized by use of adjuvant/ neoadjuvant radiotherapy or chemotherapy. For subcutaneous or intramuscular high-grade soft tissue sarcoma smaller than $5 \mathrm{~cm}$ or any size low-grade sarcoma, surgery alone should be considered if a wide excision with a good 1-2 $\mathrm{cm}$ cuff of surrounding fat and muscle can be achieved. Total laryngectomy, partial pharyngectomy and reconstruction can be done depending upon the extent of the tumour. ${ }^{10}$

There are two possible approaches: the cervical approach (lateral or anterior pharyngotomy) and endoscopic surgery .The endoscopic approach with suspension microlaryngoscopy and $\mathrm{CO} 2$ laser resection plays an important role in management. Advantages of trans-oral approach with $\mathrm{CO} 2$ laser are low or no morbidity, and patients are able to resume oral feeding within 1 or 2 days. The need for a tracheostomy is avoided and hospitalization days are decreased. Our patient resumed oral feeding in the second day and did not require a tracheostomy. He was discharged on the third postoperative day of both procedures performed in our institution. With the cervical approach, a greater number of complications may exist such as fistulas, necessity of cervical drains. Longer time to resume oral feedings and therefore, linger hospitalization. One disadvantages of laser surgery is the inability to adequately evaluate surgical margins pathologically due to fragmentation of the tumor. However, this problem will continue to be controversial regarding all laser resections for tumors, [16] However, margins were well evaluated by the surgeon experienced in laser resections. Close surveillance of the patient should be carried out every 3 months. In case or recurrence, surgical approach would be the same.

If the excision margin is close, or if there is extra muscular involvement, adjuvant radiotherapy should be added to the surgical resection to reduce the probability of local failure. However, irrespective of grade, postoperative radiotherapy is probably used more often than strictly necessary. 
Local recurrence is common, even is the long term (after 5 years). While the risk of lymph node or distant metastasis is very low. Han et $\mathrm{Ai}^{13}{ }^{13}$ In their review of literature found local recurrence in $51 \%$ of cases. Zhu et $\mathrm{aI}^{29}$ in 2017 reported four-time recurrence of tumors in one of the cases. Regional lymph node metastasis is not reported. Treatment of lymph mode areas is therefore not indicated. In case of frequent recurrence, tumor differentiation should be suspected, it has been opined that radiotherapy was only used in cases of high-grade liposarcoma (round cell and pleomorphic subtypes specifically) and not in any cases of low grade liposarcoma. Radiation therapy could play a role in inoperable progressive forms and in the event of incomplete resection. ${ }^{4}$ In a retrospective study, Longhi et $\mathrm{al}^{17}$ concluded that post-RT seems to be the best treatment for localized liposarcoma. Myxoid liposarcoma is highly radiosensitive and dramatic response with pre-operative radiation have been reported. Given the favorable outcome with wide surgical excision alone, regardless of the histologic type of the tumor, some authors believe that adjuvant therapy is unjustified.

The role of adjuvant or neoadjuvant chemotherapy is another controversial subject for liposarcomas, The majority of the randomized chemotherapy trials have shown no significant impact on overall survival; however they have found that chemotherapy does improve disease free survival, with improved local and loco-regional control. No improvement in survival has been noted when chemotherapy was added to the treatment regimen- $79 \%$ five year survival rate in the chemotherapy group versus $80 \%$ in the surgery alone group ${ }^{17}$ Preoperative use of neoadjuvant chemotherapy with anthracycline and ifosamide can be justified in selected cases with large, high-grade tumours and in certain histological types most likely to respond to chemotherapy. ${ }^{8}$ Trabectedin (Yondelis) was approved in November 2015 in the united states for unresectable or metastatic liposarcoma or leomyosarcoma who have received a prior anthracycline-containing regimen. But no improvement in survival was observed. In January 2016, the US Food and Drug Administration (FDA) approved Eribulin (Halaven) for unreachable or metastatic liposacoma in patients who received a prior anthracycline-containing regimen. Eribulin has been found to be more toxic than other drugs. ${ }^{24}$ The increasing opportunities for new therapies are based on the activation /suppression of the tumor-host immune response. ${ }^{19}$ Investigation into the efficacy of the immune response in well differentiated liposarcoma and dedifferentiated liposarcoma are ongoing and will be vital to develop new immunotherapeutic approaches to treatment.

To date, there is no reported case of distant metastasis from a laryngeal or hypopharyngeal liposarcoma. The overall prognosis is very good, with 5- year survival up to $100 \% .{ }^{29}$
The generally accepted prognostic factors for soft tissue sarcomas are size, grade and depth of the tumour, along with patient age. ${ }^{22}$ Round cell component also determine the prognosis. More than $5 \%$ of round cell carry higher risk of metastasis and recurrence. Highgrade myxoid liposarcoma carries the same prognostic information, with a greater frequency of metastasis and worse survival compared with low-grade tumors. ${ }^{14}$

Five-years disease specific survival rates (chances of not dying from cancer -related causes) are as follow: $100 \%$ in well-differentiated liposarcoma, $88 \%$ in myxoid liposarcoma and $56 \%$ in pleomorphic liposarcoma. Ten-year survival rates are $87 \%$ in well differentiated liposarcoma, $76 \%$ in lyxoid liposarcoma and $39 \%$ in pleomorphic liposarcoma. ${ }^{9}$

Well-differentiated tumors are poorly circumscribed and locally recur after incomplete excision. Although they rarely metastasize, repeated local recurrences may cause the tumor to evolve into a higher grade of sarcoma or to dedifferentiate, in which case metastasis is possible. ${ }^{24}$

\section{Conclusion}

Liposarcoma of the head and neck remains a rare occurrence, especially as a primary tumor of the aerodigestive tract. These tumors are typically misdiagnosed secondary to their indolent course and similarities in appearance to more commonly encountered benign lesions. Once identified, these tumors are best treated with wide surgical excision with a consideration for adjuvant radiotherapy. The use of chemotherapy remains controversial, and as such, further study is required to determine its effectiveness in the treatment of liposarcomas. Management of lesions of the hypo pharynx can be performed by trans oral laser surgery using suspension microlaryngoscopy. Evaluation of margins continue to be controversial in this type of management; however, these margins can and should be evaluated during surgery. Liposarcomas of the head and neck region are rare, and their management will continue to be controversial due to the low number of cases that currently exist.

Routine follow up will continue throughout the patient's life. It usually includes a physical examination and imaging studies. An MRI or CT scan of the original tumor location is typical, and a chest x-ray or CT imaging is used to detect metastasis.

\section{References}

1. Abbas Manji G, Singer S, Koff A, Schwartz GK.(2015) 4 Applicaton of molecular biology to individualize therapy for patients with liposarcoma. Am Soc Clin Oncol Educ Book 35:213-8.[Medline].

2. Adelson RT, DeFatta RJ, Verret DJ,Shen Y.(2006) Liposarcoma of the tongue: case report and review of the literature. Ear Nose Throat J 85:749-51 PubMed Am J Surg Pathol 33(5):645-58. doi:10.1097/PAS.0b013e3181963c9c. 
3. Arkun R, Memis A, Akalin T, Ustun EE, Sabah D, Kandiloglu G (1997) Liposarcoma of soft tissue: MRI findings with pathologic correlation. Skeletal Radiol 1997;26:167-72.

4. Dei Tos AP. Liposarcoma: new entities and evolving concepts. Ann Diagn Pathol 4(2000):252-

266PMID:10982304 DOI:10.1053/adpa.2000.8133

5. Doyle LA(2014) Sarcoma Classification: An Update Based on the 2013 World Health organization Classification of tumours of Soft tissue and Bone. Cancer 120(12)1763-74.

6. Faissal EI ouakif, Decourselle F, Schultheis D(2011) Liposarcoma of the hypopharynx: Two cases report and literature review Revue de laryngologie-otologierhinologie 132(4-5):245-50 Source:pubmed

7. Fletcher CDM, Unni KK, Mertens F(2002) World Health Organization Classification of Tumors. Pathology and Genetics of Tumors of soft Tissue and Bone. IARC Press, Lyon, 2002.

8. Fletcher CDM, Rydholm A, Singer S., Sundram M, Coindre JM(2002) Soft tissue tumours:epidemiology,clinical feartures,histopathological typing and grading. Pathology and Genetics of Tumors of soft Tissue and Bone. IARC Press,Lyon,2002.

9. Gebhardt M, Buecker PJ (2009) Liposarcoma: a detailed review.[Cited 2010 Jan 23]; Liddy Shriver Sarcoma Initiative 5;6-6.

10. Gleinser DM,Font JP,Cecilia G Clement CG,Mohommed BS, and UnderbrinkMP(2010)Primary myxoid liposarcoma of the supraglottic larynx Rare Tumors. Sep30;2(3):e41.

11. Golledge J, Fisher C, Evans PHR(1995) Head and neck Liposarcoma. Cancer 76(6):1051-8.

12. Graadt van Roggen Jf, Hogendoorn PC,Fletcher CD(1999) Myxoid tumours of soft tissue. Histopathol 35(4):291-312.

13. Han H, Yang L-H, Liu T-T, Wang J, Li H, Yu G, Wang Z,Lv JJ, Zhao H-y, Wang E-H(2015)Liposarcoma of the larynx:repprt of a case and review of literature. Int J Clin Exp Pathol 8(1):1068-1072.Published online 2015 Jan 1 . PMCID:PMC43488855

14. Haniball J, Sumathi VP, Kindblom LG, Abudu A,Carter SR, Tillman RM, Jeys L, Spooner D, Peake D, and Grimer RJ(2011) Prognostic Factors and Metastatic Patterns in Primary Myxoid/Round-Cell Liposarcoma. Sarcoma 2011:538085:10

15. Kanwar VS, Konstantakos AK, Dudgeon DL. Liposarcoma.[Cited 2010 Jan23]; eMedicine by WebMD $2008 ; 4(4)$.

16. Luna-Ortiz K,Campos-Ramos E, Carmona-Luna T, Mohar-Betancourt A, Ferrari-Carballo T (2009) Laser resection of liposarcoma of the hypopharynx. Med Oral Pathol Oral Cir Bucal 1;14(5):E252-6.

17. Longhi A, Neri S, Speranza C, Alberghini M, Ferrari C, Abate M, Cesari M, Ferrari S, Palmerini E, Mercuri M(2008) Liposarcoma treatment: role of radiotherapy and chemotherapy J Clin Oncol.;26

18. Mandell DL, Brandwein MS, Woo P, Som PM, Biller HF, Urken ML(1999) Upper aerodigestive Tract liposarcoma:report on four cases and literature review. Laryngoscope 109(8):1245-52. doi:10.1097/00005537199908000-00012.[PubMed][Cross Ref]

19. Nadar A. Nassif, William Tseng, Camille Borges, Peter Chen, Burton Eisenberg.(2016) Recent advances in the management of liposarcoma. Version 1.F1000Res.;5:2907. Published online 2016 Dec 22.doi:10.12688/f1---research.10050.1 PMCID:PMC5224678
20. Nouri H, Hassani R, Aderdour L, Raji A(2011) The welldifferentiated liposarcoma of the hypopharynx. Euro Ann Otorhinolaryngol Head Neck Dis 128(3):143-5. (SourceScience Direct, Elsevier)

21. Peterson JJ, Kransdort MJ, Bancroft LW, O`Connor MI(2003) Malignant fatty tumors:classification, clinical course, imaging appearance and treatment. Skeletal Radiol 32:493-503.

22. Pisters PWT, Leung DHY, Woodruff J, Shi W, and Brennan MF(1996)"Analysis of prognostic factors in 1,041 patients with localized soft tissue sarcomas of the extremities," J Clin Oncol 14(5):1679-89. [Pubmed][Google Scholar]

23. Schwartz RA, Trovato MJ, Centurion SA(2009)Liposarcoma.[Cited 2010 Jan 23]; eMedicine by WebMD. Jul;4.

24. Schwartz RA, Centurion SA(2017) Liposarcoma. emedicine.medscape.com/article/1102007-overview\#a2 Updated: May 04,eMedicine by WebMD.2017

25. Shi H.Wei L, Wang H, and Sun L(2010) Clinicopathological features of atypical liposarcoma tumors of the laryngopharynx. J Zhejiang Univ Sci B 11(12):918-22. Doi:10.1631/Jzus.B1000164

26. Wakely PE Jr.(1999) Myxomatous soft tissue tumors:correlation of cytopathology and histopathology. Ann Diagn Pathol 3(4):227-42.

27. Wenig BM, Weiss SW,Gnepp DR.(1990)Laryngeal and hypopharyngeal liposarcoma. A clinicopathologic study of 10 cases with a soft-tissue counterparts. Am J Surg Pathol 1990;14(2):134-41. doi:10.1097/00000478199002000-00005.[Pubmed] [CrossRef]doi.org/10.1016/j.anori.2010.11.006

28. Verma R,Sardana NK and Verma R(2011) Multiple Myxoid Lipomas of Supraglottic region. Int J Phonosurgery \& Larygol 1(2):88

29. Zhu H, Sun J, Wei S, Wang D, and Brandwein M(2017) Well-Differentiated Laryngeal/Hypopharyngeal Liposarcoma in the MDM2 Era Report of Three Cases and Literature Review. Head and Neck Pathol 11(2):14651.doi:10.1007/s12105-016-07447-0 